\title{
Human gut microbiota alleviate behavioural symptoms in a mouse model of autism spectrum disorder
}

Some of the core behavioural alterations in autism spectrum disorder (ASD) can be corrected by probiotic bacteria that ameliorate ASD-associated gastrointestinal (GI) dysfunction, a new study in mice suggests.

\section{Our findings...raise the exciting prospect that probiotics could be a safe and effective treatment for...autism 77}

For the study, published in Cell, Elaine Hsiao and colleagues at the California Institute of Technology (Pasadena, CA, USA) used the maternal immune activation (MIA) mouse model of ASD to assess the role of the gut-brain axis in ASD. Besides increased anxiety, stereotypical behaviour, altered sensorimotor gating, and impaired social interaction and communicationbehavioural symptoms typical of
ASD_the MIA offspring showed altered gut microbiota, increased gut permeability and elevated microbiotarelated serum metabolites. These findings are in line with studies that report a subset of individuals with ASD experience GI problems.

Hsiao and co-workers discovered that oral treatment with human commensal gut bacteria, Bacteroides fragilis, improved GI function, restored serum metabolite levels and alleviated some of the ASD-related behavioural traits-anxiety, stereotypical behaviour and sensorimotor gating-in MIA offspring mice.

GI dysfunction has been previously connected with neurological disorders such as Huntington disease and multiple sclerosis, and B. fragilis treatment has been shown to protect against neuroinflammation in a mouse model of multiple sclerosis. As yet, the involvement of gut microbiota in neurodevelopmental and behavioural disorders is poorly understood. Hsiao notes, however, that several investigations to elucidate the microbiome-gut-brain connection are under way.

"Our findings reveal that certain beneficial gut bacteria may influence behaviours relevant to neurodevelopmental disorders, and raise the exciting prospect that probiotics could be a safe and effective treatment for some cases of autism," Hsiao contends.

At the moment, the clinical benefits of therapeutics that target the microbiota in humans remain unknown, but Hsiao and colleagues hope that future trials will explore probiotic treatments for ASD and other neurodevelopmental disorders.

Hemi Malkki

Original article Hsiao, E. Y. et al. Microbiota modulate behavioral and physiological abnormalities associated with neurodevelopmental disorders. Cell doi:10.1016/ j.cell.2013.11.024 